\title{
New Electronic Interface Circuits for Humidity Measurement Based on the Current Processing Technique
}

\author{
Predrag B. Petrović ${ }^{1}$, Maria Vesna Nikolić ${ }^{2}$, Mihajlo Tatović ${ }^{1}$ \\ ${ }^{I}$ Department of Electronics, Faculty of Technical Sciences Čačak, University of Kragujevac, Svetog Save, No.65, 32000, \\ Čačak, Serbia, predrag.petroviç@ftn.kg.ac.rs \\ ${ }^{2}$ Institute for Multidisciplinary Research, University of Belgrade, Kneza Višeslava, No. 1, 11000, Belgrade, Serbia
}

\begin{abstract}
The paper describes a new electronic conditioning circuit based on the current-processing technique for accurate and reliable humidity measurement, without post-processing requirements. Pseudobrookite nanocrystalline $\left(\mathrm{Fe}_{2} \mathrm{TiO}_{5}\right)$ thick film was used as capacitive humidity transducer in the proposed design. The interface integrated circuit was realized in TSMC $0.18 \mu \mathrm{m}$ CMOS technology, but commercial devices were used for practical realization. The sensing principle of the sensor was obtained by converting the information on environment humidity into a frequency variable square-wave electric current signal. The proposed solution features high linearity, insensitivity to temperature, as well as low power consumption. The sensor has a linear function with relative humidity in the range of Relative Humidity (RH) $30-90 \%$, error below $1.5 \%$, and sensitivity $8.3 \times 10^{14} \mathrm{~Hz} / \mathrm{F}$ evaluated over the full range of changes. A fast recovery without the need of any refreshing methods was observed with a change in RH. The total power dissipation of readout circuitry was $1 \mathrm{~mW}$.
\end{abstract}

Keywords: Humidity sensor; capacity sensor interface; current-processing technique; DXCCTA; experimental verification.

\section{INTRODUCTION}

Humidity sensors have a wide application in everyday practice, including agriculture, monitoring climate change, food storage processes, and in the operation of various home appliances [1], [2]. In order to meet the demands of such applications, humidity sensors should provide high sensitivity and linearity in response, in a wide range of possible changes in processed humidity under different temperature conditions. In addition to the aforementioned demands, the sensor circuits must provide long-term stability, short response times with low energy consumption. It is preferable that a humidity sensor is compatible with standard IC components. There are different types of humidity sensors in accordance with the physical principle used to make the conversion: resistive, mechanical, gravimetric, capacitive, and thermal humidity sensors [3]. Most electronic circuits, representing the interface between the sensor itself and the processor unit, are based on the use of operational amplifiers [4]. The growing desire for miniaturization of such systems and reduction of their consumption places increasing challenges in the design process before the circuit designers. CMOS technology is a logical response to such challenges, but due to the different ratio of the width and length $(W / L)$ of the transistors used, there is a trade-off between the speed, gain, power and other parameters [5]. A solution is offered through the circuits suggested in this paper that is able to establish the best balance between the offered performances and the very strict requirements that exist in the process of designing a humidity sensor.

The sensor circuit has often been based on the principle of a resistor sensor [6]. Such sensors can detect changes in temperature, humidity, pressure, etc. The capacitive sensor on the other hand can process moisture, speed, and acoustic shift and so on. Sensing circuits that detect the change of resistance enable a relatively simple realization of the accompanying electronic interface. But if such a resistive sensor is used for moisture processing, temperature compensation must also be made, because due to the influence of temperatures under low humidity conditions, this can be difficult to detect, since the sensor circuits must have high resistance. A capacitive humidity sensor has a higher sensitivity, high reliability and low hysteresis when compared with a resistive humidity sensor. Everything listed was the reason for the development of the sensor circuit suggested in this paper.

Metal oxide semiconductor materials have been intensively investigated for application as humidity sensors [7], [8]. The humidity sensing mechanism of metal oxides is simple. It is based on water adsorption on the material surface that is composed of grains, grain boundaries, and pores. Thus, the microstructure of these materials has a 
direct influence on physical and electrical properties of these materials. Nanostructures and nanomaterials have led to many new applications of metal oxides due to changing and enhancing their microstructural properties [9]. Pseudobrookite $\left(\mathrm{Fe}_{2} \mathrm{TiO}_{5}\right)$ is an iron titanium oxide, with a band gap similar to hematite with a potential for application as a gas sensor. We have investigated a possible application of pseudobrookite for NO gas sensing [10], but our recent work has focused on humidity sensing properties of this material [11].

Analogue interface circuits used in capacitive sensors are based on the application of one of the following methods: measurement based on the application of AC sources to detect the voltage and current at unknown capacitance; using a capacitance divider; resonance and bridge circuits containing the measured capacity; methods based on charge transfer; differential methods that are able to offer high accuracy and linearity; and methods based on the application of highly sensitive capacity as a key part of the signal generator [2], [12].

This paper proposes a cheap, accurate, and reliable humidity sensor by integrating the sensing element and the conditioning circuits using standard CMOS technology for fabrication. We propose a new electronic interface circuit based on the concept of current mode processing, which is capable of converting information on environment humidity to variable frequency dependency electric current (or voltage) signals. To do this we used only one active element, DXCCTA - dual-X current conveyor transconductance amplifiers. Application of this active element is dictated by characteristics of the sensor element pseudobrookite, and its equivalent impedance circuits. Already known solutions, based on the use of some relaxation oscillators (NE555), bridge methods and integrated convertors (inside microcontrollers), cannot accurately process signals from sensors of this type [2]. The oscillation frequency, which is very high, and amplitude of the output square wave are electronically and independently controllable by the capacitance of the proposed sensor circuits, passive grounded resistance and bias current. Implementing the current mode as the base of the proposed solution offers a large dynamic range, wide bandwidth, high linearity, possibility of designing with low power consumption and simple analogue circuit design [2]. Practically, design of interface electronic circuits is the central and main contribution of the paper, because all other parts are based on known configurations, not used up until now for practical realization of a humidity sensor. DXCCTA was not used until now as the base component for realization of square-wave signals-converter of moisture to time depended current signal. We created a system for checking humidity information in real time. Moreover, the use of grounded passive components in circuit implementation is also beneficial from the integration point of view.

The proposed conditioning circuit was verified through the HSPICE simulation results carried using $0.18 \mu \mathrm{m}$ CMOS technology, and can operate very well with nonlinearity less than $1 \%$. The proposed circuit was also experimentally verified. This technology is a strong candidate for the easyto-scale implementation of next generation electronics, such as the Internet of things (IoT) [13], LoRa-based sensor technology (for example the RN2483 LoRa transceiver module), built around a Semtech SX1276 transceiver [14], and printed passive/active electronics.

Table 1. Comparison of capacitive sensor electronic interfaces circuits

\begin{tabular}{|c|c|c|c|c|c|c|}
\hline Ref. & $\begin{array}{l}\text { Type and } \\
\text { number of } \\
\text { active } \\
\text { elements }\end{array}$ & $\begin{array}{l}\text { Number of } \\
\text { passive } \\
\text { elements } \\
\text { (grou./floa.) }\end{array}$ & Verificat. & $\begin{array}{l}\text { Supply } \\
\text { voltage }\end{array}$ & Error & $\begin{array}{c}\text { Sens. }=\Delta_{\mathrm{f}} / \Delta_{\mathrm{C}} \\
\text { or } \Delta_{\mathrm{V}} / \Delta_{\mathrm{C}}\end{array}$ \\
\hline$[13]$ & $1 \mathrm{CFOA}$ & $10(5 / 5)$ & sim./exp. & $\pm 1.65 \mathrm{~V}$ & $\begin{array}{l}< \pm 12 \% \\
< \pm 5 \% \\
\end{array}$ & $\begin{array}{l}247 \times 10^{3} \mathrm{~V} / \mathrm{F} \\
912 \times 10^{3} \mathrm{~V} / \mathrm{F} \\
\end{array}$ \\
\hline$[16]$ & $\begin{array}{c}3 \text { (trans. } \\
\text { stage, diff. } \\
\text { and } \\
\text { curr.amp.) }\end{array}$ & $6(4 / 2)$ & sim./exp. & $2.5 \mathrm{~V}$ & $\pm 1.5 \%$ & $1.33 \times 10^{11} \mathrm{~V} / \mathrm{F}$ \\
\hline$[17]$ & $2 \mathrm{CCII}$ & $5(1 / 4)$ & sim./exp. & $\begin{array}{l} \pm 5 \mathrm{~V}- \\
\pm 15 \mathrm{~V}\end{array}$ & $<5 \%$ & $2.6 \times 10^{10} \mathrm{~Hz} / \mathrm{F}$ \\
\hline [18] & $1 \mathrm{CCII}$ & $3(2 / 1)$ & sim./exp. & $\pm 10 \mathrm{~V}$ & $\mathrm{~N} / \mathrm{A}$ & $1.6 \times 10^{14} \mathrm{~Hz} / \mathrm{F}$ \\
\hline [19] & $4 \mathrm{CCII}$ & $5(2 / 3)$ & sim./exp. & $\pm 10 \mathrm{~V}$ & $<3 \%$ & $1.1 \times 10^{12} \mathrm{~V} / \mathrm{F}$ \\
\hline$[20]$ & 2 CCII & $5(4 / 1)$ & sim./exp. & $\mathrm{N} / \mathrm{A}$ & $\mathrm{N} / \mathrm{A}$ & $2.1 \times 10^{10} \mathrm{~Hz} / \mathrm{F}$ \\
\hline$[21]$ & $\begin{array}{c}4 \text { (MLT, } \\
\text { OA) }\end{array}$ & $8(3 / 5)$ & exp. & N/A & $\pm 0.8 \%$ & N/A \\
\hline$[22]$ & $3 \mathrm{CCII}$ & $6(5 / 1)$ & sim./exp. & $\pm 6 \mathrm{~V}$ & N/A & $3 \times 10^{10} \mathrm{~Hz} / \mathrm{F}$ \\
\hline$[23]$ & $1 \mathrm{CCII}$ & $4(1 / 3)$ & sim./exp. & $\pm 1.2 \mathrm{~V}$ & $< \pm 10 \%$ & $\begin{array}{c}4.2 \times 10^{10} \\
\mathrm{~Hz} / \mathrm{F}\end{array}$ \\
\hline$[24]$ & 1 DVCC & $3(2 / 1)$ & sim./exp. & $\pm 15 \mathrm{~V}$ & N/A & $1 \times 10^{10} \mathrm{~Hz} / \mathrm{F}$ \\
\hline$[25]$ & $\begin{array}{l}\text { 1 DO- } \\
\text { DVCC }\end{array}$ & $3(3 / 0)$ & sim./exp. & $\pm 10 \mathrm{~V}$ & $\mathrm{~N} / \mathrm{A}$ & $8 \times 10^{13} \mathrm{~Hz} / \mathrm{F}$ \\
\hline$[26]$ & $2 \mathrm{CCII}$ & $4(3 / 1)$ & sim./exp. & $\pm 9 \mathrm{~V}$ & $<7 \%$ & $2 \times 10^{11} \mathrm{~Hz} / \mathrm{F}$ \\
\hline$[27]$ & $\begin{array}{c}5 \mathrm{OA} \\
\text { (capac. } \\
\text { bridge and } \\
\text { relax. osc.) }\end{array}$ & $12(0 / 12)$ & exp. & $\pm 15 \mathrm{~V}$ & $<3 \%$ & $1 \times 10^{13} \mathrm{~Hz} / \mathrm{F}$ \\
\hline$[28]$ & $\begin{array}{l}9 \text { OA (imp. } \\
\text { to vol. } \\
\text { conv.) }\end{array}$ & $18(3 / 15)$ & exp. & $\pm 9 \mathrm{~V}$ & $\pm 1.6 \%$ & $1 \times 10^{11} \mathrm{~V} / \mathrm{F}$ \\
\hline $\begin{array}{l}\text { This } \\
\text { work }\end{array}$ & 1 DXCCTA & $1(1 / 0)$ & sim./exp. & $\pm 1.25 \mathrm{~V}$ & $\begin{array}{c}< \pm 1.5 \\
\% \\
\end{array}$ & $\begin{array}{c}8.3 \times 10^{14} \\
\mathrm{~Hz} / \mathrm{F}\end{array}$ \\
\hline
\end{tabular}

A comparison of various electronic interfaces and transducers used for capacitive sensors, which are known and verified through available literature, is given in Table 1. The proposed solutions were estimated based on their main characteristics and parameters - from the viewpoint of the direct relation to capacitance sensing and transduction. Compared to the most up-to-date and previously known solutions, the originality and main contributions of the proposed interface circuit here are reflected in the fact that a new simplified CMOS topology of the active element was developed, which is used to generate a square electric current signal with variable frequency and defined amplitude-current mode output; realization requires a minimum number of passive elements - only one, which is grounded, which is an absolute priority in realization in integrated technology. In addition, the realized sensor system offers high accuracy and sensitivity in operation, low consumption, which ensures the system's autonomy over a longer period of time, with much lower overall complexity. Also, the proposed interface circuit generates an autonomous frequency dependent current output signal waveform, and contrary to all the mentioned methods in Table 1., does not require additional control logic and postprocessing, an external clock signal, synchronism, and switching mechanism. The generated frequency variable electric current signal on the output of the proposed circuits can be directly used as a measure of environment humidity. The proposed circuit, based on simulation tests, consumes a 
power of $1 \mathrm{~mW}$, which enables its long-lasting autonomy in operation (battery powered systems, IoT operation). Unfortunately, the circuits used for the comparison (Table 1.) with the solution proposed here do not provide consumption data (no explicit measurements of the consumption have been performed in any of the papers), so it was not possible to make a comparison on that basis. If we make estimation based on the catalogue data for the components used in their realization (Table 1.), we can conclude that all mentioned solutions require much more power for operation - more than $10 \mathrm{~mW}$.

\section{Proposed ELECTRONIC INTERFACE CIRCUITS}

We used a newly proposed active element, DXCCTA as the base for the realization of the interface between the transducer and acquisition unit (for example, microcontroller PIC 18F45K80, a high performance 8-bit MCU [28]), Fig.1.a). Practically, DXCCTA is a combination of two active elements: DXCCII and an operational transconductance amplifier (OTA), with electronic tunability capability [29], enjoying all the benefits of DXCCII and OTA - but they have never been used in realization of a sensor circuit. Some applications require an extra buffer in the active element to meet the requirement of an appropriate impedance level for the output signal [12], [29]. This is not the case with our proposed sensor circuits, because we select port connections in a new and appropriate way, in a different way from the configuration proposed in [29]. This approach gave us the opportunity to throw out the additional buffer stage that exists in [29], and significantly simplify the interface circuit. Fig.1.b) shows CMOS implementation of the used DXCCTA [29], without extra buffer stage. As we can see, DXCCTA has eight terminals of which $x$ and $x$-terminals are low impedance terminals, whereas the terminals $y, z+, z-, o_{1+}, o_{2-}$, and $o_{3+}$ are high impedance terminals. In CMOS implementation of DXCCTA, Fig.1.b), MOS transistors $\mathrm{M}_{1}-\mathrm{M}_{20}$ form DXCCII, and MOS transistors $\mathrm{M}_{21}-\mathrm{M}_{32}$ form OTA stages.

The terminal characteristics of DXCCTA is described in equation (1), where $g_{m 1}, g_{m 2}$, and $g_{m 3}$ are the transconductances of OTA1 $\left(\mathrm{M}_{21}, \mathrm{M}_{22}, \mathrm{M}_{27}, \mathrm{M}_{28}\right)$, OTA2 $\left(\mathrm{M}_{23}, \mathrm{M}_{24}, \mathrm{M}_{29}, \mathrm{M}_{30}\right)$, and OTA3 $\left(\mathrm{M}_{25}, \mathrm{M}_{26}, \mathrm{M}_{31}, \mathrm{M}_{32}\right)$, respectively [29].

$$
\begin{aligned}
& v_{x+}=v_{y}, v_{x-}=-v_{y}, i_{z+}=i_{x+}, i_{z-}=i_{x-} \\
& i_{o 1+}=g_{m 1} v_{z-}, i_{o 2-}=-g_{m 2} v_{z-}, v_{o 3+}=g_{m 3} v_{z-}
\end{aligned}
$$

The transconductances, $g_{m i}$ in equation (1) depend on the bias currents $I_{B i}(i=1,2,3)$ and are expressed as $g_{m i}=\sqrt{k_{n i} I_{B i}}$ where $k_{n i}=\mu_{n} C_{O X}(W / L)_{i}$ is the physical parameter of MOS transistors. It is well known that the physical parameter, $k_{n i}$ is temperature dependent thus, the transconductance, $g_{m i}$ is also temperature dependent and its value decreases with increase in temperature.

If MOS transistors $\left(\mathrm{M}_{21}-\mathrm{M}_{32}\right)$ of OTA stages are operated in the saturation region and PMOS transistors $\left(\mathrm{M}_{21}-\mathrm{M}_{26}\right)$ forming the current mirrors are perfectly matched then the currents at $o_{1+}, o_{2-}$, and $o_{3+}$ terminals $\left(i_{o 1^{+}}, i_{o 2-}\right.$, and $i_{o 3+}$, respectively) are given as [29]

$$
\begin{aligned}
& i_{o 1+}=I_{B 1} ; i_{o 2-}=-I_{B 2} ; i_{o 3+}=I_{B 3} \text { for } v_{z-}=V_{D D} \\
& i_{o 1+}=-I_{B 1} ; i_{o 2-}=I_{B 2} ; i_{o 3+}=-I_{B 3} \text { for } v_{z-}=V_{S S}
\end{aligned}
$$

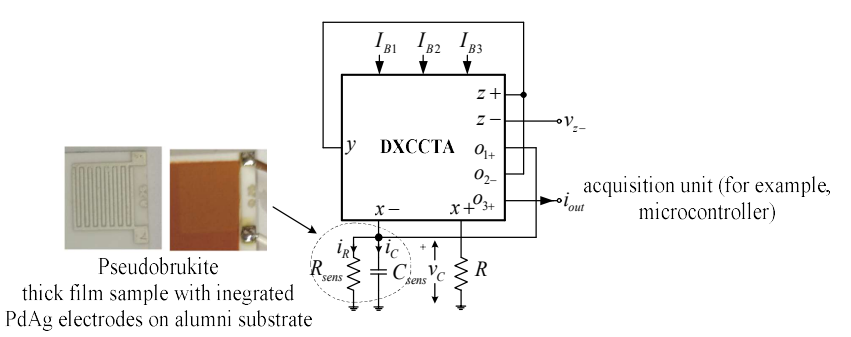

a)

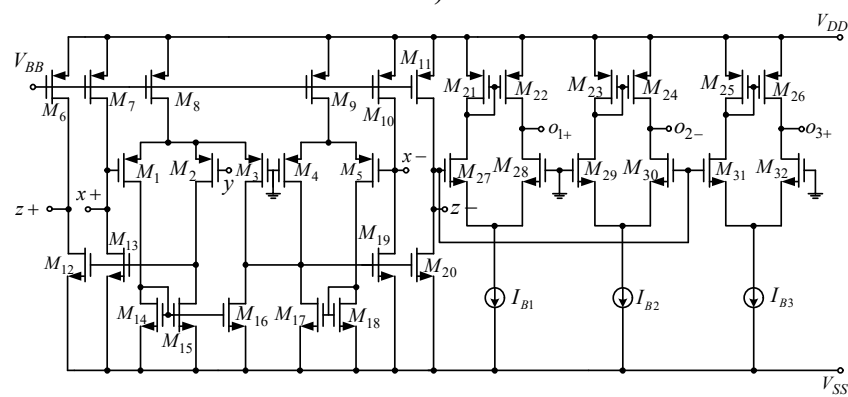

b)

Fig.1. a) Circuit of the proposed conditioning-interface circuits, b) CMOS implementation of DXCCTA.

The proposed humidity to frequency converter (Fig.1.a)) comprises a single DXCCTA, one grounded resistor and grounded humidity transducer, which we can equivalently represent as parallel connection of resistor and capacitor, $R_{\text {sens }}$ and $C_{\text {sens }}$ (transducer equivalent impedances). Fig.1.a) shows that terminal $z$ - is floated, therefore the voltage, $v_{Z \text { - }}$ saturates either to $V_{D D}$ or $V_{S S}$ depending on the current $i_{z-}$ [29]. The voltage, $v_{z-}$ in saturation mode is expressed as follows:

$$
v_{z-}=\left\{\begin{array}{l}
V_{D D} \text { for } i_{z-} \geq 0 \\
V_{S S} \text { for } i_{z-}<0
\end{array}\right.
$$

These voltage levels are high enough to saturate all OTAs and electric currents. For these reasons, currents $i_{o 1+}, i_{o 2-}$, and $i_{o 3+}$ become totally dependent on bias currents of their respective OTA stage as defined in (2). The two saturation levels of $i_{\text {out }}$ are $I_{B 3}$ and $-I_{B 3}$. The duration of intervals in which this square wave current signal follows these saturation levels depends on the voltage dynamics across the sensor equivalent circuit. The threshold levels are $V_{H T}$ (higher-upper threshold) and $V_{L T}$ (lower threshold), and they are defined with a bias current $I_{B 2}$ and external resistance $R$. We can suppose that initially $i_{\text {out }}$ is at its positive saturation level, $I_{B 3}$. At the same time $i_{o 1+}$ and $i_{o 2-}$ will be $I_{B 1}$ and $-I_{B 2}$, respectively. The current, $i_{o 1+}$ causes the capacitor to charge with the following dynamics (due to the different nature of 
the load introduced by the sensor circuit related to port $x$-, the dynamics of change of the observed quantities is completely different in regards to relations that were described in [29]):

$$
\begin{aligned}
& i_{\text {Csens }}(t)=\frac{v_{C \text { Sens }}(t)}{R_{\text {sens }}}+C_{\text {sens }} \frac{d v_{\text {Csens }}(t)}{d t}, 0 \leq t \leq T_{O N} \\
& \Rightarrow R_{\text {sens }} C_{\text {sens }} \frac{d v_{\text {Csens }}(t)}{d t}+v_{\text {Csens }}(t)=I_{B 1} R_{\text {sens }}
\end{aligned}
$$

The limiting values of voltage across the sensor were defined with the threshold level $V_{H T}$ of $v_{x+}$. When $v_{x+}$ becomes just higher than the level $V_{H T}$, the sum of currents $i_{z+}$ and $i_{o 2-}$ becomes negative causing $v_{z-}$ to saturate to $V_{S S}$. Therefore, $i_{\text {out }}$ is now changed to $-I_{B 3}$ from positive saturation level, $I_{B 3}$. Thus, $i_{o 1+}$ and $i_{o 2-}$ are now changed to $I_{B 1}$ and $I_{B 2}$, respectively. A negative current, $-I_{B 1}$ at terminal, $o_{1+}$ causes the sensor to discharge with the above defined dynamics until $v_{x+}$ reaches the threshold level $V_{L T}$. When $v_{c s e n s}$ becomes just less than $V_{L T}$, the sum of currents $i_{z^{+}}$and $i_{\text {o2 }}$ becomes positive causing $v_{z \text { - }}$ to saturate to $V_{D D}$ and $i_{\text {out }}$ is again changed to $I_{B 3}$. The amplitude of $i_{\text {out }}$ is expressed as

$$
i_{\text {out }}=\left\{\begin{array}{l}
I_{B 3} \text { for } v_{z-}=V_{D D} \\
-I_{B 3} \text { for } v_{z-}=V_{S S}
\end{array}\right.
$$

The threshold levels of $v_{x+}$ and the peak to peak amplitude, $v_{x+(p-p)}$ are given as

$$
\begin{aligned}
& V_{H T}=I_{B 2} R \text { and } V_{L T}=-I_{B 2} R \\
& v_{x+(p-p)}=V_{H T}-V_{L T}=2 I_{B 2} R
\end{aligned}
$$

The on and off time periods ( $T_{O N}$ and $T_{O F F}$, respectively) are obtained from voltage across the sensor (their waveforms) by comparing the slope during these two time periods:

$$
\begin{aligned}
& v_{\text {Cens }}(t)=I_{B 1} R_{\text {sens }}\left(1-e^{-1 / \tau}\right)+V_{T L} e^{-1 / \tau}, \tau=R_{\text {sens }} C_{\text {sens }} \\
& \Rightarrow V_{T H}=I_{B 1} R_{\text {sens }}\left(1-e^{-T_{\text {oN }} / \tau}\right)+V_{T L} e^{-T_{O N} / \tau} \\
& \Rightarrow T_{O N}=\tau \ln \frac{V_{T L}-I_{B 1} R_{\text {sens }}}{V_{T H}-I_{B 1} R_{\text {sens }}}=\tau \ln \frac{I_{B 2} R+I_{B 1} R_{\text {sens }}}{I_{B 1} R_{\text {sens }}-I_{B 2} R}
\end{aligned}
$$

During the off time period, the voltage across the sensor will be changed with the following dynamics:

$$
\begin{aligned}
& R_{\text {sens }} C_{\text {sens }} \frac{d v_{\text {Csens }}(t)}{d t}+v_{\text {Cens }}(t)=-I_{B 1} R_{\text {sens }}, T_{O N} \leq t \leq T_{O N}+T_{O F F}=T \\
& \Rightarrow v_{\text {Csens }}(t)=I_{B 1} R_{\text {sens }}\left(e^{-\left(t-T_{O N}\right) / \tau}-1\right)+V_{T H} e^{-\left(t-T_{O N}\right) / \tau}, v_{\text {Csens }}\left(T_{O N}+T_{O F F}\right)=V_{T L} \\
& \Rightarrow T_{O F F}=\tau \ln \frac{V_{T H}+I_{B 1} R_{\text {sens }}}{V_{T L}+I_{B 1} R_{\text {sens }}}=\tau \ln \frac{I_{B 2} R+I_{B 1} R_{\text {sens }}}{I_{B 1} R_{\text {sens }}-I_{B 2} R}
\end{aligned}
$$

From (8), it is noted that both cycle periods are equal thus, the duty cycle of the generated square electric current signal on port $o_{3+}$ is fixed to $50 \%$. The oscillation frequency, $f_{0}$ is obtained from $T_{O N}$ and $T_{O F F}$ as follows

$$
f_{o}=\frac{1}{T_{\text {ON }}+T_{\text {OFF }}}=\frac{1}{2 R_{\text {sens }} \mathrm{C}_{\text {sens }} \ln \frac{I_{B 2} R+I_{B 1} R_{\text {sens }}}{I_{B 1} R_{\text {sens }}-I_{B 2} R}}
$$

On the base of (5) and (9), we can conclude that the amplitude of $i_{\text {out }}$ and oscillation frequency, $f_{0}$ are electronically and independently tunable via bias currents, $I_{B 3}$ and $I_{B 1}$, respectively. Also, the period of the generated square wave current output signal directly depends on parameters of sensor circuits. This way we come into the position to indirectly recalculate the humidity of the environment in which we place our sensor from information on the generated frequency of the electric current output signal. The generated output signal is completely autonomous and its frequency has no effect on the capacitance of the sensor, which is very often seen in the so far known interface circuit realizations [26], in which the detection of moisture is based on the principle of moisture adsorption and desorption.

The performance of the proposed circuits can be further evaluated based on the sensitivity of its response relative to the sensor parameters $C_{\text {sens }}$ and $R_{\text {sens }}$. Sensitivity $(S)$ is defined as an incremental change in the output signal value relative to the incremental change in the sensor parameter [30]. According to such a criterion, the sensitivity of the analysed circuits for the interface is obtained as

$$
\begin{aligned}
& S_{C_{\text {sens }}}=\frac{\partial f_{0}}{\partial C_{\text {sens }}}=-\frac{1}{2 R_{\text {sens }} C_{\text {sens }}^{2} \ln \frac{I_{B 2} R+I_{B 1} R_{\text {sens }}}{I_{B 1} R_{\text {sens }}-I_{B 2} R}} \\
& S_{R_{\text {sens }}}=\frac{\partial f_{0}}{\partial R_{\text {sens }}}=\frac{\frac{\left.I_{B 2} R+I_{B 1} R_{\text {sens }}\right)\left(I_{B 1} R_{\text {sens }}-I_{B 2} R\right)}{2 I_{\text {sens }}}-\ln \frac{I_{B 2} R+I_{B 1} R_{\text {sens }}}{I_{B 1} R_{\text {sens }}-I_{B 2} R}}{2 R_{\text {sens }}^{2} C_{\text {sens }} \ln ^{2} \frac{I_{B 2} R+I_{B 1} R_{\text {sens }}}{I_{B 1} R_{\text {sens }}-I_{B 2} R}}
\end{aligned}
$$

Based on the obtained relations (10), the sensitivity can be adjusted by properly selecting the values of the parameters, and for measured values (sections 4 and 5 of paper) we can conclude that the proposed circuits offer satisfactory high sensitivity.

\section{A. Humidity transducer}

The pseudobrookite humidity transducer was developed by screen printing thick film paste on alumina substrate with test interdigitated $\mathrm{PdAg}$ electrodes. This design is simple and is commonly applied for sensing [31]. Pseudobrookite powder and thick film paste was synthesized and characterized in detail and this is described [11]. Interdigitated $\mathrm{PdAg}$ electrodes were first screen printed on alumina substrate and fired in a conveyer furnace at $850{ }^{\circ} \mathrm{C}$ for 10 minutes in air [11]. The electrode dimensions were: width $8 \mathrm{~mm}$, length $8 \mathrm{~mm}$, electrode spacing $0.25 \mathrm{~mm}$ (Fig.1.a)). Five layers of pseudobrookite thick film paste were then screen printed on the substrate with electrodes, with the procedure described in detail in [11] achieving a porous nanocrystalline thick film layer about $60 \mu \mathrm{m}$ thick (as each layer was $\sim 12 \mu \mathrm{m}$ ).

The influence of the change in relative humidity $(\mathrm{RH}) 30$ - 
$90 \%$ of several thick film pseudobrookite sensors on complex impedance were monitored in a humidity chamber and analysed in detail in [11]. The response and recovery times were relatively rapid (16s) and relatively low hysteresis (difference between absorption and desorption) were obtained showing that pseudobrookite thick film sensors are good candidates for application in humidity sensing.

\section{ESTIMATION OF THE INFLUENCES OF NON-IDEALITIES AND PARASITIC EFFECTS ON MEASUREMENT ACCURACY}

In a non-ideal case the terminal characteristics of DXCCTA can be described as [29]

$$
\begin{aligned}
& v_{x+}=\beta_{1} v_{y}, v_{x-}=-\beta_{2} v_{y}, i_{z+}=\alpha_{1} i_{x+}, i_{z-}=\alpha_{2} i_{x-}, \\
& i_{o 1+}=\gamma_{1} g_{m 1} v_{z-}, i_{o 2-}=-\gamma_{2} g_{m 2} v_{z-}, i_{o 3+}=\gamma_{3} g_{m 3} v_{z-}
\end{aligned}
$$

Also, in a non-ideal case, the currents, $i_{o 1+}, i_{o 2-}$, and $i_{o 3+}$ are modified as follows:

$$
\begin{aligned}
& i_{o 1+}=\alpha_{3} I_{B 1} ; i_{o 2-}=-\alpha_{4} I_{B 2} ; i_{o 3+}=\alpha_{5} I_{B 3} \text { for } v_{z-}=V_{D D} \\
& i_{o 1+}=-\alpha_{3} I_{B 1} ; i_{o 2-}=\alpha_{4} I_{B 2} ; i_{o 3+}=-\alpha_{5} I_{B 3} \text { for } v_{z-}=V_{S S}
\end{aligned}
$$

where, $\alpha_{1}, \alpha_{2}, \alpha_{3}, \alpha_{4}$, and $\alpha_{5}$ are the non-ideal current transfer gains from $i_{x+}$ to $i_{z+}, i_{x-}$ to $i_{z-}, I_{B 1}$ to $i_{o 1_{+}}, I_{B 2}$ to $i_{o 2-}$, and $I_{B 3}$ to $i_{o 3+}$, respectively; $\beta_{1}$ and $\beta_{2}$ are non-ideal voltage transfer gains from $v_{y}$ to $v_{x+}$, and $v_{y}$ to $v_{x-}$, respectively; $\gamma_{1}, \gamma_{2}$, and $\gamma_{3}$ are transconductance inaccuracies from $v_{z-}$ to $i_{o 1+}, v_{z-}$ to $i_{o 2-}$, and $v_{z-}$ to $i_{o 3+}$, respectively [29].

Taking into account the parasitic impedances involved in DXCCTA, when analysing the proposed sensor circuit it must be assumed that they exist: resistances $R_{x+}$ and $R_{x-}$ at $x+$ and $x$ - terminals, whereas the parallel combinations of $\left(R_{y} / /\left(1 / \mathrm{s} C_{y}\right)\right),\left(R_{z+} / /\left(1 / \mathrm{s} C_{z^{+}}\right)\right),\left(R_{z} / /\left(1 / \mathrm{s} C_{z-}\right)\right),\left(R_{o 1_{+}} / /\left(1 / \mathrm{s} C_{o 1+}\right)\right)$, $\left(R_{o 2-} / /\left(1 / \mathrm{s} C_{o 2-}\right)\right)$, and $\left(R_{o 3+} / /\left(1 / C_{o 3+}\right)\right)$ appear at $y, z+, z-, o_{1+}$, $o_{2-}$ and $o_{3+}$ terminals - Fig.1.a), respectively [29]. For the CMOS implementation of DXCCTA in Fig.1.b), we measured the parasitic impedances of the terminals (using simulation in HSPICE programme), in order to evaluate their effects on processing capabilities of the proposed interface circuits. The values of these parasitic elements are given in Table 2.

Table 2. Simulated values of parasitic components, i.e. the parasitic impedances of DXCCTA.

\begin{tabular}{|c|c|}
\hline Parasitic & \begin{tabular}{c} 
Simulated Values \\
\hline$R_{y}, C_{y}$
\end{tabular} \\
\hline $\begin{array}{c}1932 \times 10^{12} \Omega, 2.45 \\
\mathrm{fF}\end{array}$ \\
\hline$R_{x+}$ & $132 \Omega$ \\
\hline$R_{x-}$ & $285 \Omega$ \\
\hline$R_{z+}, C_{z^{+}}$ & $30.2 \mathrm{k} \Omega, 4.38 \mathrm{fF}$ \\
\hline$R_{z-}, C_{z-}$ & $30.3 \mathrm{k} \Omega, 13 \mathrm{fF}$ \\
\hline$R_{o 1^{+}}, C_{o 1^{+}}$ & $58.2 \mathrm{k} \Omega, 3.29 \mathrm{fF}$ \\
\hline$R_{o 2-}, C_{o 2-}$ & $58.2 \mathrm{k} \Omega, 3.31 \mathrm{fF}$ \\
\hline$R_{o 3+}, C_{o 3+}$ & $58.2 \mathrm{k} \Omega, 3.35 \mathrm{fF}$ \\
\hline
\end{tabular}

Taking the above described non-idealities and parasitic impedances into consideration, the proposed conditioning circuit is reanalysed. The amplitude of the output squarewave electric current $i_{\text {out }}$ becomes defined as follows

$$
i_{\text {out }}=\left\{\begin{array}{l}
\alpha_{5} I_{B 3} \text { for } v_{z-}=V_{D D} \\
-\alpha_{5} I_{B 3} \text { for } v_{z-}=V_{S S}
\end{array}\right.
$$

while threshold levels and peak to peak amplitude are given as

$$
\begin{gathered}
V_{H T}=\frac{\alpha_{4} I_{B 2} R^{\prime}}{\alpha_{1}} ; V_{L T}=-\frac{\alpha_{4} I_{B 2} R^{\prime}}{\alpha_{1}} \\
V_{x+(p-p)}=V_{H T}-V_{L T}=\frac{2 \alpha_{4} I_{B 2} R^{\prime}}{\alpha_{1}}
\end{gathered}
$$

where, $R^{\prime}=R+R_{X+}$. The oscillation frequency is also modified

$$
f_{o}=\frac{1}{T_{O N}+T_{\text {OFF }}}=\frac{1}{2 R_{\text {sens }} \mathrm{C}^{\prime} \ln \frac{\alpha_{4} I_{B 2} R^{\prime}+\alpha_{1} \alpha_{3} I_{B 1} R_{\text {sens }}}{\alpha_{1} \alpha_{3} I_{B 1} R_{\text {sens }}-\alpha_{4} I_{B 2} R^{\prime}}}
$$

where, $C^{\prime}=C_{\text {sens }}+C_{\text {ol+ }}$. On the base of the above equations (13)-(15), we can conclude that non-idealities and the parasitic impedances of DXCCTA affect the amplitude of $i_{\text {out }}$, threshold levels, peak to peak amplitude, and oscillation frequency, respectively. The effects of these non-idealities could be neglected depending upon the operating frequency range of the proposed sensor circuits, because non-ideal gains deviate from unity only at higher frequencies. Also, parasitic effects can be minimized by a proper choice of $R$ and sensor parameters $\left(C_{\text {sens }}\right.$ and $\left.R_{\text {sens }}\right)-R$ must be selected such that $R \gg R_{x+}$ and the capacitor, $C_{\text {sens }}$ must be chosen such that $C_{\text {sens }} \gg>C_{o 1+}$. Correct selection of the observed parameters leads to improvement in the dynamic range of the sensor circuit, with reduction in the effect of the parasitic components. This is achieved by increasing the value of only one element-grounded resistive load, which must be greater than the parasitic resistance on the same terminal of the DXCCTA. In addition, it is possible to adjust the sensitivity of the circuit and the frequency band through external passive components, as well as the change of bias currents.

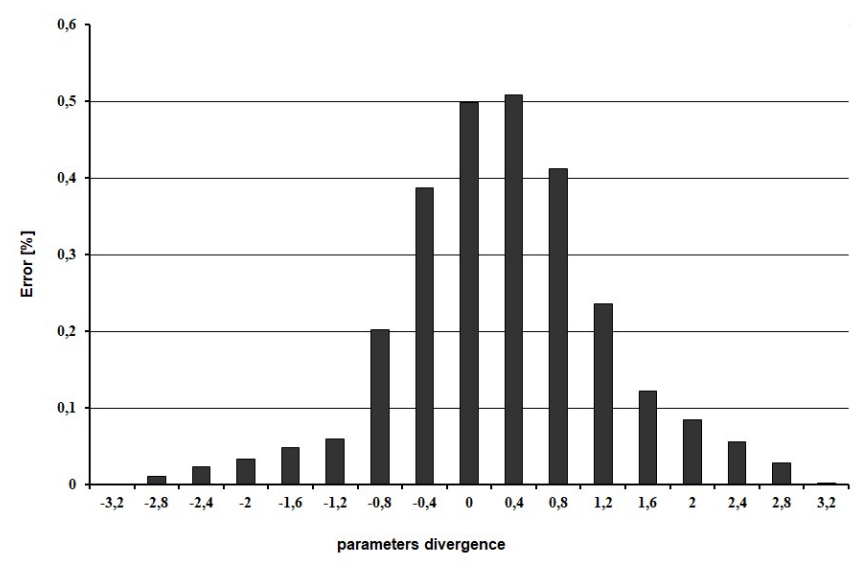

Fig.2. Distribution of errors in the behaviour of the proposed interface circuit, for divergence in the value of parameters, from their nominal values. 
In order to further check the performance of the proposed interface circuit, in a situation where there is variation in the fabrication process - the production of semiconductor elements and voltage variation, Monte Carlo simulation (provided by the HSPICE software package itself) was performed in 1000 runs. During this analysis, the voltage supply of $\pm 1.25 \mathrm{~V}$, bias voltage of $0.42 \mathrm{~V}$, and bias current of $50 \mu \mathrm{A}$ amplitude were used, which resulted in the histogram in Fig.2. It is assumed that due to possible variation in the manufacturing process, the threshold voltage of all MOS transistors deviates by $5 \%$ (Gaussian deviation) and that the variation in the supply voltage $\left(V_{D D}\right.$ and $\left.V_{S S}\right)$ is the order of $5 \%$ (Gaussian deviation). We assumed that the extreme PVT (Process Voltage Temperature) variations were in the range of $+/-5 \%$ (this tolerance is applied over the $0{ }^{\circ} \mathrm{C}$ to $100{ }^{\circ} \mathrm{C}$ temperature range).

On the basis of such conducted analysis, we are in a position to investigate the effect of the process parameters and the mismatch between transistors on the precision of processing. We define the lower and upper limits of the interval, which contains $95 \%$ of error - absolute value of difference between the predicted and observed output value [32]. The standard deviation in the generated output current input signal was approximately $0.64 \mu \mathrm{A}$. It was noted that such changes do not lead to a larger deviation in the frequency of the generated current signal (order of $2 \%$ ) and that the amplitudes of the output waveforms are not disturbed.

\section{DESIGN PROCEDURE AND SIMULATION RESULTS}

Simulations were performed using HSPICE with $0.18 \mu \mathrm{m}$ TSMC CMOS process parameters. The supply voltages of $\pm 1.25 \mathrm{~V}$, and bias voltage, $V_{B B}=0.42 \mathrm{~V}$ were used in the simulation. The aspect ratios ( $W / L$ ratios) of MOS transistors used in the CMOS implementation of DXCCTA are given in Table 3.

Table 3. MOS transistor aspect ratios $(W / L)$.

\begin{tabular}{|c|c|}
\hline $\mathrm{M}_{1}-\mathrm{M}_{2}$ & $0.72 / 0.36$ \\
\hline $\mathrm{M}_{3}-\mathrm{M}_{5}$ & $1.44 / 0.36$ \\
\hline $\mathrm{M}_{14}-\mathrm{M}_{15}$ & $1.34 / 0.36$ \\
\hline $\mathrm{M}_{16}-\mathrm{M}_{18}$ & $2.4 / 0.36$ \\
\hline $\mathrm{M}_{6}-\mathrm{M}_{13}, \mathrm{M}_{19}-\mathrm{M}_{20}$ & $4.8 / 0.36$ \\
\hline $\mathrm{M}_{21}-\mathrm{M}_{26}$ & $1.44 / 0.36$ \\
\hline $\mathrm{M}_{27}-\mathrm{M}_{32}$ & $3.6 / 0.36$ \\
\hline
\end{tabular}

The resistance $R$ in Fig.1.a) is chosen to be $1 \mathrm{k} \Omega$ ( $R>>R_{X^{+}}$-specified in Table 2.). For the specified values of magnitude of $i_{\text {out }}=50 \mu \mathrm{A}, v_{C(p-p)}=100 \mathrm{mV}$, the values of $I_{B 1}$, $I_{B 2}, I_{B 3}$ are found according to the above conducted analysis as follows: $I_{B 1}=I_{B 2}=I_{B 3}=50 \mu \mathrm{A}$ and $C_{\text {sens }}=500 \mathrm{pF}, R_{\text {sens }}=$ $4 \mathrm{M} \Omega$. The simulated transient responses of the output square-wave electric current $i_{\text {out }}$ and voltage across sensor element $v_{C}$ are shown in Fig.3.a). The obtained oscillation frequency of $0.5 \mathrm{MHz}$ is similar to the calculated value. In Fig.3.b), the transient responses of observed values, when $I_{B 1}$ is changed to $80 \mu \mathrm{A}$, are shown. The simulated frequency is now changed to $0.789 \mathrm{MHz}(1 \%$ error $)$ at $I_{B 1}=80 \mu \mathrm{A}$. It is obvious that the threshold levels of voltage $v_{C}$ and amplitude levels of output electric current $i_{\text {out }}$ are not affected by the change in bias current, $I_{B 1}$. Fig.3.c) demonstrates the possibility of electronic control of the amplitude of the generated current signal $i_{\text {out }}$ for two different values of $I_{B 3}\left(40 \mu \mathrm{A}\right.$ and $60 \mu \mathrm{A}, I_{B 1}=40 \mu \mathrm{A}$, $I_{B 2}=60 \mu \mathrm{A}$ ). The amplitude of $i_{\text {out }}$ is independently tunable without affecting the oscillation frequency. Dependencies of $f_{0}$ on $C_{\text {sens }}$ are shown in Fig.3.d). A significant influence on the accuracy of the generated $f_{0}$ starts from $C_{\text {sens }}<400 \mathrm{pF}$.

The bandwidth of the realized interface circuit is limited by the frequency of the humidity transducer used, which, at this frequency range, retains the desired characteristics. DXCCTA has a much broader bandwidth, which is determined by the simulation and is $20 \mathrm{MHz}$.

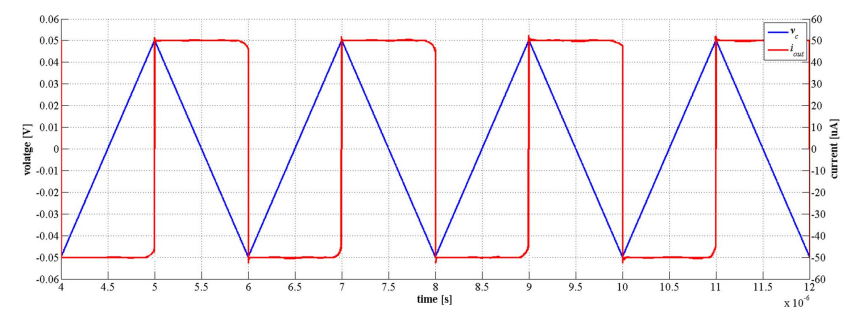

a)

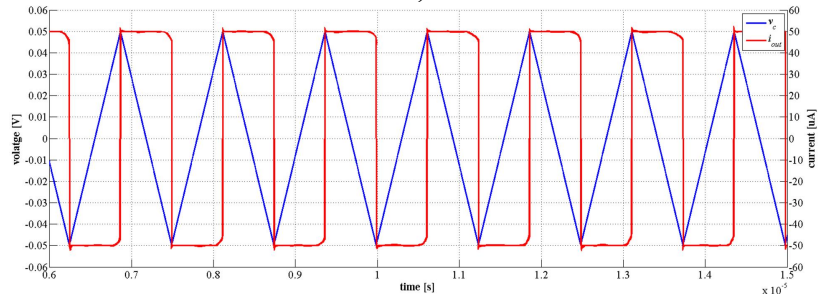

b)

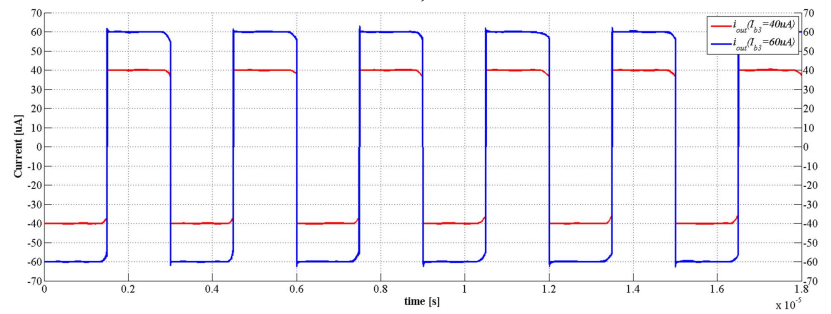

c)

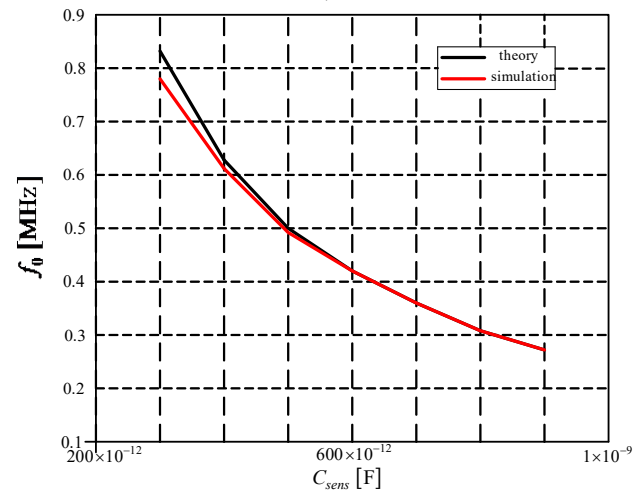

d)

Fig.3. Transient response of proposed interface circuits a) Simulated waveforms of $i_{\text {out }}$ and $v_{C}, I_{B I}=I_{B 2}=I_{B 3}=50 \mu \mathrm{A}, f_{o}$ $=0.5 \mathrm{MHz}, \mathrm{b})$ simulated waveforms of $i_{\text {out }}$ and $v_{C}, I_{B I}=80 \mu \mathrm{A}$, $\left.I_{B 2}=I_{B 3}=50 \mu \mathrm{A}, f_{o}=0.789 \mathrm{MHz}, \mathrm{c}\right)$ Electronic tuning of amplitude of $i_{\text {out }}$ for different values of $I_{B 3}(40 \mu \mathrm{A}$ and $60 \mu \mathrm{A})$, (d) $f_{0}$ versus $C_{\text {sens }}, I_{B I}=I_{B 2}=I_{B 3}=50 \mu \mathrm{A}$. 
From the Fig.3.a) and Fig.3.b) we can see that voltage across the sensor possesses a virtually linear characteristic that results from the value of its time constant, which leads to very fast voltage fluctuations in the observed boundaries. Among other things, the proposed conditioning and conversion circuits show the sensitivity of $8.3 \times 10^{14} \mathrm{~Hz} / \mathrm{F}$ (Fig.3.d)), which is much better than other circuits used for comparison in Table 1.

\section{EXPERIMENTAL MEASUREMENT RESULTS}

The possible practical implementation of DXCCTA using commercial ICs (AD844 as a current feedback operational amplifier and LM13700) is shown in Fig.4. [29], [33] (DXTCCTA is not commercially available, and authors are not currently in a position to realize it in the form of IC). The experiments were carried out using $\pm 5 \mathrm{~V}$ supply voltages. The transconductance value of LM13700 at bias current value of $100 \mu \mathrm{A}$ is $1.5 \mathrm{mS}$. The bias currents were set to $I_{\mathrm{B} 1}=I_{\mathrm{B} 2}=I_{\mathrm{B} 3}=140 \mu \mathrm{A}$ and passive components used are: $R=3 \mathrm{k} \Omega, R_{a}=R_{b}=10 \mathrm{k} \Omega$, on the basis of the evaluation conducted in Sections 2 and 3 of this paper.

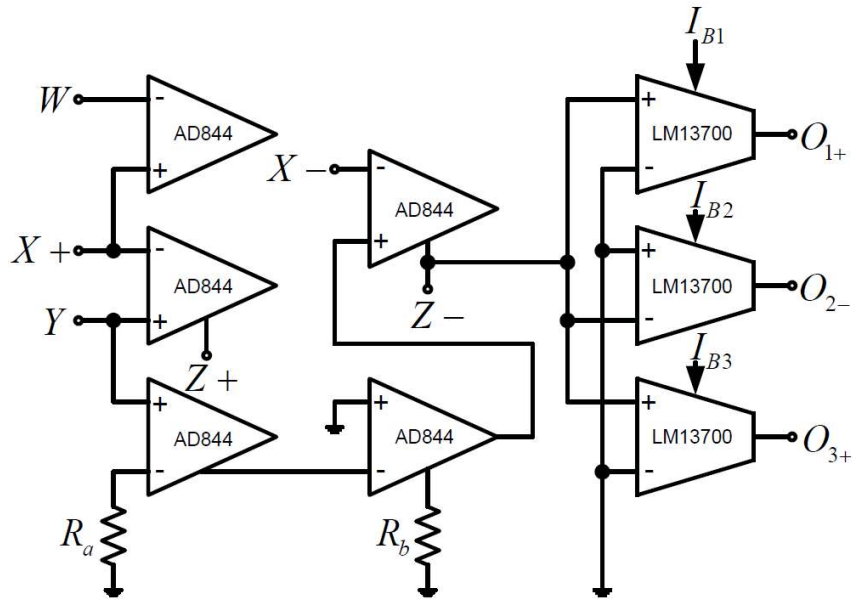

Fig.4. Practical implementation of multiple outputs DXCCTA based on commercially available ICs.

During the experimental checks, the measured values of voltage and electric current using the TDS20114 oscilloscope (2500 samples) on the interface circuits were first recorded and then transferred to the MATLAB environment, without altering to plot the curves.

For experimental verification of the proposed design we used a JEIO TECH TH-KE-025 temperature and humidity climatic chamber in the relative humidity range 30-90\% [11]. Prior to each measurement the samples were dried/heated for 20 minutes at $50{ }^{\circ} \mathrm{C}$ to remove any moisture. The test sample was placed into the chamber and using wires soldered to the electrodes we established a connection with our interface. The humidity was varied between 30 and $90 \%$ at $25^{\circ} \mathrm{C}$, by setting the desired humidity value (it took on average 45-90 minutes to establish the desired humidity value, i.e. saturation to be reached). The experimentally observed waveforms of $i_{\text {out }}$ and $v_{x+}$ are shown in Fig.5. - the response in the time domain of the proposed sensor system in a situation where the environmental humidity is changed. The experimental oscillation frequency in situation when moisture is $60 \% \mathrm{RH}$ is $0.1542 \mathrm{MHz}(0.58 \%$ error in comparison with calculated frequency). Fig.5.a) and Fig.5.b) proves the high sensitivity of the proposed electronic circuit and its ability to accurately process the detected change in the humidity of the environment in which the sensor system is located. The electronic tuning of amplitude of $i_{\text {out }}$ via bias current $I_{\mathrm{B} 3}=$ $250 \mu \mathrm{A}$ is shown in Fig.5.c), which shows that the amplitude of $i_{\text {out }}$ can be independently controlled without disturbing the oscillation frequency.

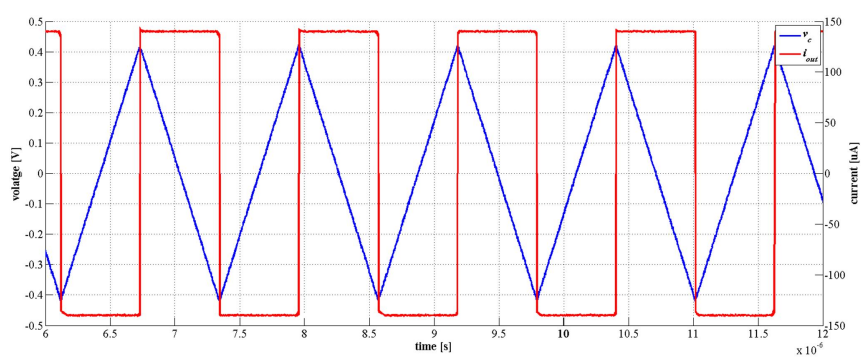

a)

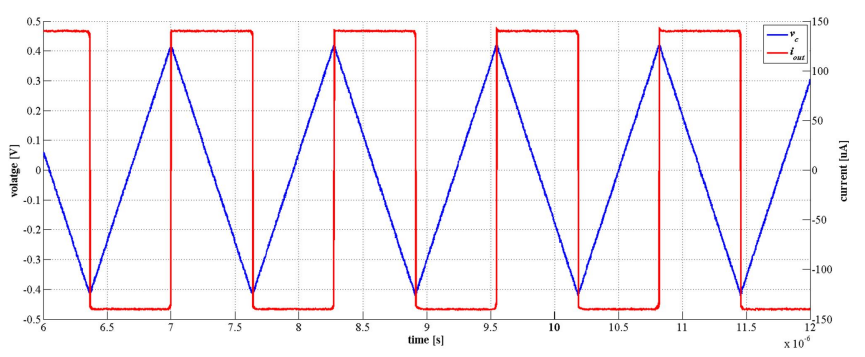

b)

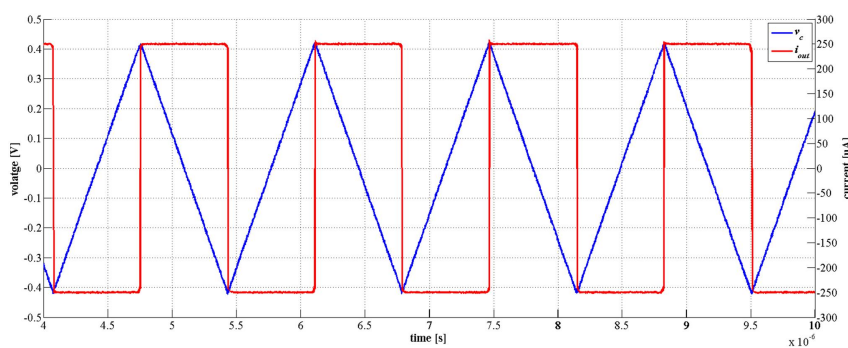

c)

Fig.5. The time domain behaviour of the proposed interface circuits a) $30 \% \mathrm{RH}$, b) $60 \% \mathrm{RH}$, c) $90 \% \mathrm{RH}$ and bias current $I_{B 3}$ $=250 \mu \mathrm{A}$.

Using the MATLAB environment we detected the frequency of the output electric current signal and subsequent signal conditioning (on external resistance connected to the input pin of the acquisition card to obtain a voltage equivalent). To count input edges of the square wave output signal, the timer as a counter has been used. The dependence of the frequency $f$ of the output electric current signal on the humidity of the environment in which the sensor circuit is located (the chamber used during the experimental performance test of the proposed sensor system) is shown in Fig.6. It shows that the frequency is proportional to the capacitance change with moisture 
content, and follows the nature of the capacitance (impedance) change observed in [11]. In the same Fig.6., we compared the simulation and experimental measurement results. In order to obtain simulation results for comparison, we used data on the values of the equivalent sensor circuit $C_{\text {sens }}$ and $R_{\text {sens }}$, which are given in [11], as a function of humidity, after which a simulation check was performed as described in Section 4 of the paper. We can see that the obtained results are well correlated because the error (relative) in the operating range of $30 \%$ to $90 \% \mathrm{RH}$ is below $0.65 \%$. The proposed sensor system possesses satisfactory properties in terms of error and gives a linear frequency change relationship. Over the observed full scale, the frequency change with the moisture is nonlinear (the square-wave electric current output signal), which is due to the nonlinear capacitive response of the sensor [11].

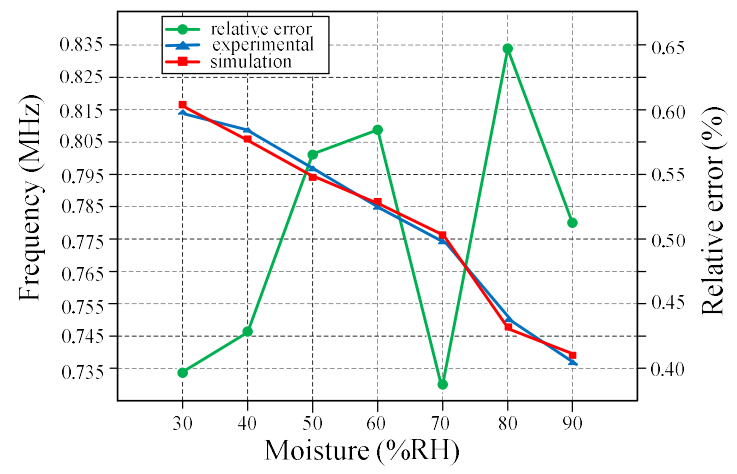

Fig.6. Features of the proposed interface circuits-change in the frequency of the output signal with moisture concentration and difference (error) between experimental data and expectationsimulation results (theory versus measurement).

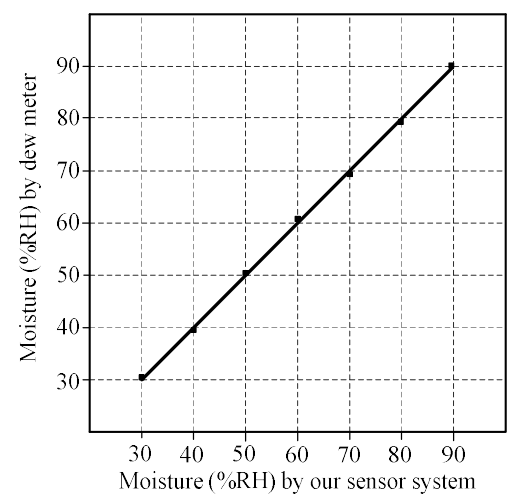

Fig.7. Comparison of the meter output with a commercial dew point meter.

The response time of a sensor when exposed to moisture is defined as the time in which a sensor reaches $90 \%$ of the total response, while recovery is the time required for a sensor to return to $90 \%$ of the original baseline signal, when moisture is removed [11]. The average response time was about $16 \mathrm{~s}$, while the recovery time was very fast and the sensor recovered in $1 \mathrm{~s}$. The difference in response and recovery times was attributed to the microstructure of pseudobrookite thick films that represented a porous network of aggregated nanoparticles [11]. Internal porosity was relatively low compared to the number and volume of pores between agglomerates, so water vapours were not trapped making desorption when the sensor was removed from the climatic chamber undisturbed as water vapours exited the porous voids between agglomerates.

The lookup table stored in the memory is used for calibrating the oscillation frequency in terms of ppm. The frequency sensitivity and the nonlinearity of the sensor for the $30-90 \% \mathrm{RH}$ moisture range are found to be $8.1 \times 10^{14}$ $\mathrm{Hz} / \mathrm{F}$ and $\sim 1 \%$, respectively. Measurement results (transferred to a PC) were compared with those measured by a commercial dew point meter and the error has been estimated. The accuracy of the moisture meter with the laboratory capacitive sensor for the specified range of moisture $30-90 \% \mathrm{RH}$ is nearly $\pm 1.5 \%$, when compared with the commercial meter (accuracy $0.1 \%$ ) [34] - Fig.7.

\section{CONCLUSION}

In this paper, a humidity sensor read-out circuitry using DXCCTA has been designed. The proposed interface provides a simple interconnection with the associated processing unit without post-processing, with very low power consumption of $1 \mathrm{~mW}$. It is important to note that the proposed design can be fully realized in the form of an integrated circuit. The proposed solution is based on generating a fully autonomous electric current signal, the period of which is linearly dependent on the capacity of the humidity sensor. The possibility of precise humidity measurement in the range of $30 \%$ to $90 \% \mathrm{RH}$ with error less than $1.5 \%$ was experimentally confirmed (sensitivity $8.3 \times 10^{14} \mathrm{~Hz} / \mathrm{F}$ over the full range of changes). In addition, the available bias currents and external resistor can be used for slight correction of the operation of the proposed concept. To the best of author's knowledge, such a solution for the capacitance to frequency interface has not been proposed and experimentally verified in previous presented work, while achieving fairly good performance in terms of both accuracy and dynamic range. The square wave output offers immunity to parasitic capacitance and low frequency noise ( $1 / f$ and $\mathrm{dc}$ offset). The design can be used for a humidity sensor and can be adopted by industries due to its flexibility in design that could be beneficial from the point of view of industrial production costs.

\section{ACKNOWLEDGMENT}

This work was supported in part by the projects OI 172057, 42009 and III45007, funded by the Ministry of Education, Science and Technological Development of the Republic of Serbia.

\section{REFERENCES}

[1] Reverter, F., Casas, O. (2008). Direct interface circuit for capacitive humidity sensors. Sensors and Actuators A, 143, 315-322. https://doi.org/10.1016/j.sna.2007.11.018. 
[2] Islam, T. (2017). Advanced interfacing techniques for the capacitive sensors. In Advanced Interfacing Techniques for Sensors. Springer, SSMI 25, p. 73-109. https://doi.org/10.1007/978-3-319-55369-6_2.

[3] Kuriyal, N., Kumar, R., Ramola, V. (2014). Optimization and Simulation of humidity sensor readout circuitry using two stage op amp. IOSR Journal of Electrical and Electronics Engineering, 9 (5), 66-72. https://doi.org/10.9790/1676-09536672.

[4] Jalkanen, T., Määttänen, A., Mäkilä, E., Tuura, J., Kaasalainen, M., Lehto, V.P., Ihalainen, P., Peltonen, J., Salonen, J. (2015). Fabrication of porous silicon based humidity sensing elements on paper. Journal of Sensors, 2015, art. ID 927396.

https://doi.org/10.1155/2015/927396.

[5] Nizhnik, O., Higuchi, K., Maenaka, K. (2014). A standard CMOS humidity sensor without postprocessing. Sensors, 11 (6), 6197-6202.

https://doi.org/10.3390/s110606197.

[6] Nath, P., Hussain, I., Dutta, S., Choudhury, A. (2014). Solvent treated paper resistor for filter circuit operation and relative humidity sensing. Indian Journal of Physics, 88 (10), 1093-1097.

https://doi.org/10.1007/s12648-014-0547-x.

[7] Blank, T.A., Eksperiandorova, L.P., Belikov, K.N. (2016). Recent trends of ceramic humidity sensors development. Sensors and Actuators B, 228, 416-442. https://doi.org/10.1016/j.snb.2016.01.015.

[8] Urrutia, A., Goicoechea, J., Ricchiuti, A.L., Barrera, V.D., Sales, M.S., Arregui, F.J. (2016). Simultaneous measurement of humidity and temperature based on a partially coated optical fiber long period grating. Sensors and Actuators B, 227, 135-141. https://doi.org/10.1016/j.snb.2015.12.031.

[9] Mirzaei, A., Hashemi, B., Janghorban, K. (2016). $\alpha$ $\mathrm{Fe} 2 \mathrm{O} 3$ based nanomaterials as gas sensors. Journal of Materials Science: Materials in Electronics, 27, 31093144. https://doi.org/10.1007/s10854-015-4200-z.

[10] Miskovic, G., Lukovic, M.D., Nikolic, M.V., Vasiljevic, Z.Z., Nicolics, J., Aleksic, O.S. (2016). Analysis of electronic properties of pseudobrookite thick films with a possible application for NO gas sensing. In Proceedings of the 39th International Spring Seminar on Electronics Technology, 2016, 386391. DOI: 10.1109/ISSE.2016.7563226.

[11] Nikolic, M.V., Vasiljevic, Z.Z., Lukovic, M.D., Pavlovic, V.P., Vujancevic, J., Radovanovic, M., Krstic, J.B., Vlahovic, B., Pavlovic, V.B. (2018). Humidity sensing properties of nanocrystalline pseudobrookite (Fe2TiO5) based thick films. Sensors and Actuators B, 277, 654-664. https://doi.org/10.1016/j.snb.2018.09.063.

[12] Polak, L., Sotner, R., Petrzela, J., Jerabek, J. (2018). CMOS current feedback operational amplifier-based relaxation generator for capacity to voltage sensor interface. Sensors, 18 (12), 4488. https://doi.org/10.3390/s18124488

[13] Islam, T., Mukhopadhyay, S.C., Suryadevara, N.K. (2017). Smart sensors and internet of things: A postgraduate paper. IEEE Sensors Journal, 17 (3), 577-584. DOI: 10.1109/JSEN.2016.2630124.

[14] Ameloot, T., Torre, P.V., Rogier, H. (2018). A compact low-power LoRa IoT sensor node with extended dynamic range for channel measurements. Sensors, 18 (7), 2173. https://doi.org/10.3390/s18072137.

[15] Scotti, G., Pennissi, S., Monsurro, P., Trifiletti, A. (2014). $88-\mu$ A $1-\mathrm{MHz}$ stray-insensitive CMOS current-mode interface IC for differential capacitive sensors. IEEE Transactions on Circuits and Systems I, 61 (7), 1905-1916. DOI: 10.1109/TCSI.2014.2298275.

[16] Pal, D., Srinivasulu, A., Pal, B.B., Demosthenous, A., Das, B.N. (2009). Current conveyor-based square/triangular waveform generators with improved linearity. IEEE Transactions on Instrumentation and Measurement, 58 (7), 2174-2180. DOI: 10.1109/TIM.2008.2006729.

[17] Abuelma'atti, M.T., Al-Absi, M.H. (2005). A current conveyor based relaxation oscillator as versatile electronic interface for capacitive and resistive sensors. Int. Journal of Electronics, 92 (8), 473-477. https://doi.org/10.1080/08827510410001694798.

[18] Ferri, G., Parente, F.R., Stornelli, V. (2017). Current conveyor-based differential capacitance analog interface for displacement sensing application. $A E U$ International Journal of Electronics and Communications, 81, 83-91.

https://doi.org/10.1016/j.aeue.2017.07.014.

[19] Almashary, B., Alhokail, H. (2000). Current-mode triangular wave generator using CCIIs. Microelectronics Journal, 31 (4), 239-243. https://doi.org/10.1016/S0026-2692(99)00106-8.

[20] Depari, A., Sisinni, E., Flammini, A., Ferri, G., Stornelli, V., Barile, G., Parente, F.R. (2018). Autobalancing analog front end for full-range differential capacitive sensing. IEEE Transactions on Instrumentation and Measurement, 67 (4), 885-893. DOI: 10.1109/TIM.2017.2785160.

[21] Srinivasulu, A. (2012). Current conveyor based relaxation oscillator with tunable grounded resistor/capacitor. International Journal of Design, Analysis and Tools for Integrated Circuits and Systems, 3 (2).

[22] Marcellis, A.D., Ferri, G., Mantenuto, P. (2017). A CCII-based non-inverting Schmitt trigger and its application as a stable multivibrator for capacitive sensor interfacing. International Journal of Circuit Theory and Applications, 45 (8), 1060-1076. https://doi.org/10.1002/cta.2268.

[23] Chien, H.C. (2013). Design and implementation of relaxation generators: New application circuits of the DVCC. International Journal of Electronics, 100 (2), 227-244.

https://doi.org/10.1080/00207217.2012.687193. 
[24] Chien, H.C. (2013). Square/triangular wave generator using single DO-DVCC and three grounded passive components. American Journal of Electrical and Electronic Engineering, 1 (2), 32-36.

https://doi.org/10.12691/ajeee-1-2-3.

[25] Malik, S., Kishore, K., Artee, S.A., Akbar, T., Islam, T. (2016). A CCII-based relaxation oscillator as a versatile interface for resistive and capacitive sensors. In 3rd International Conference on Signal Processing and Integrated Networks (SPIN). IEEE, 359-363. DOI: 10.1109/SPIN.2016.7566719.

[26] Khan, A.U., Islam, T., Akhtar, J. (2016). An oscillatorbased active bridge circuit for interfacing capacitive sensors with microcontroller compatibility. IEEE Transactions on Instrumentation and Measurement, 65 (11), 2560-2568. DOI: 10.1109/TIM.2016.2581519.

[27] Khan, A.U., Islam, T., George, B., Rehman, M. (2019). An efficient interface circuit for lossy capacitive sensors. IEEE Transactions on Instrumentation and Measurement, 68 (3), 829-836. DOI: 10.1109/TIM.2018.2853219.

[28] Microchip Technology Inc. (2017). Microchip PIC $16(L) F 19155$.
[29] Chaturvedi, B., Kumar, A. (2019). Fully electronically tunable and easily cascadable square/triangular wave generator with duty cycle adjustment. Journal of Circuits, Systems and Computers, 28 (6), 1950105. https://doi.org/10.1142/S0218126619501056.

[30] Amico, A.D., Natale, C.D. (2001). A contribution on some basic definitions of sensors properties. IEEE Sensors Journal, 1 (3), 183-190. DOI: 10.1109/JSEN.2001.954831.

[31] Fine, G.F., Cavanagh, L.M., Afonja, A., Binions, R. (2010). Metal oxide semi-conductor gas sensors in environmental monitoring. Sensors (Basel), 10 (6), 5469-5502. https://doi.org/10.3390/s100605469.

[32] Joint Committee for Guides in Metrology. (2008). Evaluation of measurement data - Guide to the expression of uncertainty in measurement, $1 \mathrm{st}$ edition. JCGM 100:2008.

[33] Maheshwari, S., Ansari, M.S. (2012). Catalog of realizations for DXCCII using commercially available ICs and applications. Radioengineering, 21 (1), 281289.

[34] SHAW Moisture Meters Ltd. (2004). Shaw automatic dewpoint meter data manual.

Received September 30, 2020

Accepted February 26, 2021 